\title{
The Clinical and Technical Effectiveness of Endovascular Embolization in Patients with Nonvariceal Gastrointestinal Bleeding
}

\author{
Azadeh Elmi $^{1} \quad$ T. Gregory Walker ${ }^{1} \quad$ Suvranu Ganguli ${ }^{1}$ Sanjeeva P. Kalva ${ }^{2}$
}

\author{
${ }^{1}$ Massachusetts General Hospital, Boston, Massachusetts, United States \\ 2 University of Texas Southwestern Medical Center, Dallas, Texas, \\ United States
}

Address for correspondence Sanjeeva P. Kalva, MD, University of Texas Southwestern Medical Center, 5323 Harry Hines Blvd, Dallas, TX 75390-8834 (e-mail: Sanjeeva.Kalva@UTSouthwestern.edu).

J Clin Interv Radiol ISVIR 2017;1:5-12.

\begin{abstract}
Keywords

- gastrointestinal bleeding

- endoscopy

- angiography

- embolization

Background and Aim Endovascular embolization is a well-established option in the management of acute gastrointestinal bleeding (GIB) after failed therapeutic endoscopy; however, questions remain concerning the outcomes and the various predictors of clinical and technical success of this therapy. The authors aimed to assess the effectiveness of endovascular embolization in patients with nonvariceal GIB.

Method Clinical records of 88 patients (mean age: 67.8 years) who underwent endovascular embolization for GIB were reviewed. Patient demographics, history, angiographic findings, treatment, and outcomes were recorded. The technical success of embolization, and the 24-hour and 30-day rebleeding and mortality rates were calculated. Multivariate analysis was performed to assess the factors associated with 24-hour and 30-day rebleeding.

Results Angiography demonstrated signs of bleeding in 63 (71.6\%) patients and all underwent selective embolization of the abnormal artery. Empiric embolization was performed in 25 patients. Embolization was performed with coils $(n=45)$, Gelfoam $(n=12)$, microparticles $(n=14)$, glue $(n=2)$, or a combination of these $(n=15)$. The technical success rate was $96.6 \%$. The 24 -hour and 30-day rebleeding occurred in 13 $(14.7 \%)$ and $16(18.2 \%)$ patients, respectively. The 24 -hour and 30-day mortality rates were 9.1 and $11.3 \%$, respectively. Ischemic complications following embolization were seen in three patients, of which two required surgery. Based on the multivariate analysis, the need for continued transfusion after embolization and prior GIB were independent variables associated with 24-hour and 30-day rebleeding, respectively.

Conclusions Endovascular embolization has a high technical and clinical success in patients presenting with nonvariceal GIB.
\end{abstract}

\section{Introduction}

Gastrointestinal bleeding (GIB) is a common problem that accounts for approximately $2 \%$ of hospital admissions. ${ }^{1}$ Although factors such as response to fluid resuscitation and/or transfusion, hypotension, the presence of shock, and coagulopathy are determinants of appropriate treatment strategy, ${ }^{2}$ the majority of nonvariceal GIB resolves spontaneously. ${ }^{3}$ When bleeding persists, endoscopy is the main- stay for diagnosis and treatment and results in primary hemostasis in the majority of the cases. However, in a subset of patients, endoscopic therapy fails to halt bleeding, necessitating angiographic evaluation and transcatheter arterial embolization or surgery for bleeding control. ${ }^{4}$ In patients who are poor surgical candidates or in those with an unclear source of bleeding, angiography cannot only provide information about the location and cause of bleeding but may allow therapeutic intervention to stop the 
bleeding through embolization or regional vasopressor infusion. ${ }^{5-7}$

Although endovascular embolization is a well-established option in the management of acute upper and lower GIB after failed therapeutic endoscopy, ${ }^{8-10}$ questions remain concerning the outcomes and the various predictors of clinical and technical success of this therapy. In this study, we aimed to assess the clinical and technical effectiveness of endovascular embolization in patients with nonvariceal GIB.

\section{Subjects and Methods}

\section{Study Cohort}

Institutional Review Board approval was obtained for this retrospective single-center study that was undertaken in compliance with the Health Insurance Portability and Accountability Act. Because the study was retrospective and limited to review of medical records, the requirement for informed consent from subjects was waived. We identified patients who underwent angiography and embolization for persistent GIB despite medical management (which included intravenous fluids, transfusion of blood products, and acid-suppressing medication) at our institution from the database (Hi-IQ Society of Interventional Radiology, ConexSys, Lincoln, RI) maintained by the interventional radiology service. Patients with variceal bleeding were excluded.

\section{Angiography and Embolization}

Fellowship-trained interventional radiologists performed the angiography and embolization through common femoral arterial access. Selective celiac, superior mesenteric, and inferior mesenteric arteriography and arterioportography were performed with a $5 \mathrm{~F}$ visceral catheter (Cobra, Sos, Mikaelsson, and Simmons, Cook Medical Inc., Bloomington, IL) and 12 to $35 \mathrm{~mL}$ of a nonionic contrast material injected at a rate of 3 to $6 \mathrm{~mL} / \mathrm{s}$. During the procedure, the $5 \mathrm{~F}$ catheter or a coaxially placed $3 \mathrm{~F}$ microcatheter (Renegade, Boston Scientific, Natick, MA; Progreat, Terumo, Japan) was advanced selectively into suspected branch vessels for superselective angiography. Embolization was performed through the $3 \mathrm{~F}$ microcatheter when active bleeding and/or pseudoaneurysm were identified on angiography. The embolic material was selected based on the type of vessel being embolized and the preference of the radiologist. The size of embolic agent varied from 500 to $900 \mu \mathrm{m}$. Empiric embolization of the branch vessel supplying the site of upper GIB localized by endoscopy was performed in those cases in which there was no active extravasation/pseudoaneurysm demonstrated by angiography. For example, for a lesion in the stomach, left gastric artery was embolized, whereas for a duodenal lesion, gastroduodenal artery was embolized.

\section{Data Collection}

Clinical information including admission vital signs, comorbidities (diabetes mellitus, stroke, cardiovascular disease, and renal disease), use of anticoagulants, alcohol use, prior GIB, and transfusion requirements were derived from patients' charts. Endoscopic and imaging reports were reviewed to identify the etiology, anatomical location, and possible arterial feeder of GIB. Procedural data pertaining to embolization procedure were collected. This information included the site of embolization, the presence or absence of active extravasation, pseudoaneurysm and mass, the materials used for embolization, and postprocedure complications such as bleeding, ischemia, hematoma, or thrombosis. The technical and clinical success, and 24-hour and 30-day rebleeding and mortality rates were calculated for upper and lower GIB and were compared. All outcomes were examined until May 30, 2012. Multivariate analysis was performed to assess the factors associated with 24-hour and 30-day rebleeding. Clinical success was defined as cessation of GIB with no additional need for other hemostatic interventions including endoscopic intervention or surgery. Technical success of embolization was defined as complete occlusion of the target vessel on postembolization angiography.

\section{Statistical Analysis}

Statistical software (SPSS, version 17.0, SPSS Inc.) was used for analysis. The Fisher exact test was used to evaluate categorical data. Multivariate Cox proportional hazard analysis was performed for age, site of bleeding (upper versus lower), comorbidities, use of anticoagulant, history of prior GIB, materials used for embolization, and red blood cell (RBC) transfusion within 12 hours after the embolization to determine their relationship with rebleeding at 24 hours and 30 days after embolization.

\section{Results}

A total of 88 patients (61 men and 27 women) with a mean age of 67.8 years (range: 22-94) were included in this study. Sixty-two patients had upper GIB and 26 had lower GIB. Computed tomography (CT) angiography was performed in 34 patients, and 47 (54.7\%) patients underwent endoscopy immediately prior to angiography. All endoscopies were positive and demonstrated active bleeding site $(65.4 \%$ patients with upper GIB and 34.6\% of patients with lower GIB). Tc-99m labeled RBC scan was available in nine (10.2\%) patients and was positive for bleeding in seven. The most common comorbidities were cardiovascular disease $(n=49$; $55.7 \%)$ and renal disease ( $n=17 ; 19.3 \%)$. Fourteen (15.9\%) patients were using anticoagulants at the time of bleeding, while coagulopathy (defined as international normalized ratio [INR] $>1.5$ times control and/or partial thromboplastin time $>44$ seconds) prior to embolization was noted in 31 ((35.2\%) patients. The range of INR in our patients with anticoagulation abnormality was 1.6 to 4.7 and the mean INR value was 2.07 . Thirty (34.1\%) patients had a history of prior alcohol use and 41 (46.6\%) patients had a history of prior GIB. The cause of GIB was determined in 77 patients (-Table 1). The most common causes were malignancy (19.3\%), iatrogenic (16\%), and duodenal ulcer (16\%). The iatrogenic cases included post-Whipple procedure in seven, after liver biopsy in two, after abdominal aortic aneurysm repair in two, after ovarian cancer surgery in one, after band ligation for hemorrhoids in one, and bleeding from colectomy 
Table 1 Patient characteristics

\begin{tabular}{|c|c|}
\hline Characteristics & No. of patients (\%) \\
\hline \multicolumn{2}{|l|}{ Sex } \\
\hline Male & $61(69.3 \%)$ \\
\hline Female & $27(30.7 \%)$ \\
\hline \multicolumn{2}{|l|}{ Age group } \\
\hline Mean & 67.8 \\
\hline Range & $23-94$ \\
\hline \multicolumn{2}{|l|}{ Presenting symptom } \\
\hline Hematemesis & $16(18 \%)$ \\
\hline Hematochezia & $18(20.5 \%)$ \\
\hline Melena & $8(9.1 \%)$ \\
\hline Hematemesis and melena & $5(5.7 \%)$ \\
\hline $\begin{array}{l}\text { Postprocedural bleeding } \\
\text { (through a drain or postbiopsy) }\end{array}$ & $7(8 \%)$ \\
\hline $\begin{array}{l}\text { CT findings after } \\
\text { trauma or surgery }\end{array}$ & $5(5.7 \%)$ \\
\hline Hypotension and weakness & $16(18 \%)$ \\
\hline Abdominal pain & $13(14.8 \%)$ \\
\hline \multicolumn{2}{|c|}{ Hemoglobin concentration before angiography } \\
\hline$<8$ & $21(23.86 \%)$ \\
\hline $8-10$ & $51(58 \%)$ \\
\hline$>10$ & $16(18.2 \%)$ \\
\hline \multicolumn{2}{|l|}{ Coagulopathy } \\
\hline INR $>1.5$ & $31(35.27 \%)$ \\
\hline PTT $>44 \%$ & $13(14.7 \%)$ \\
\hline \multicolumn{2}{|l|}{ Comorbidities $^{\text {a }}$} \\
\hline 0 & $27(30.6 \%)$ \\
\hline 1 & $18(20.5 \%)$ \\
\hline$>1$ & 43 (49\%) \\
\hline
\end{tabular}

Abbreviations: $\mathrm{CT}$, computed tomography; INR, international normalized ratio; PTT, partial thromboplastin time.

${ }^{a}$ Comorbidities included diabetes mellitus, stroke, cardiovascular disease, and renal disease.

site in one. Among the 88 patients, 60 were unstable requiring intensive care unit admission.

Positive angiographic findings were present in 63 (71.6\%) patients, including active contrast material extravasation in 46 , pseudoaneurysm in 11 , and both in 6 ; these patients underwent selective arterial embolization. Stomach and duodenum were the most common sites of bleeding followed by liver. The most commonly identified source of arterial bleeding/pseudoaneurysm was the gastroduodenal artery $(n=17)$ followed by the hepatic artery $(n=15)$ and colic arteries $(n=8)$ (-Table 2). In $25(28.4 \%)$ patients with upper GIB, no contrast material extravasation or pseudoaneurysm was identified and subsequently empiric embolization was performed based on the bleeding site identified on endoscopy or CT angiography findings. The overall technical success rate of embolization was $96.6 \%$ (85 of 88
Table 2 Causes and locations of bleeding

\begin{tabular}{|c|c|}
\hline Factors & No. of patients (\%) \\
\hline \multicolumn{2}{|l|}{ Causes of bleeding } \\
\hline Malignancy & $17(19.3 \%)$ \\
\hline latrogenic & $14(16 \%)$ \\
\hline Duodenal ulcer & $14(16 \%)$ \\
\hline Trauma & $9(10.2 \%)$ \\
\hline Diverticulum & $9(10.2 \%)$ \\
\hline Gastric ulcer & $6(6.8 \%)$ \\
\hline Arteriovenous malformation & $2(2.2 \%)$ \\
\hline Pseudoaneurysm & $2(2.2 \%)$ \\
\hline Dieulafoy's lesion & $1(1.1 \%)$ \\
\hline \multicolumn{2}{|l|}{ Site of bleeding } \\
\hline Stomach and duodenum & 24 \\
\hline Liver & 15 \\
\hline Colon & 9 \\
\hline Spleen & 3 \\
\hline $\begin{array}{l}\text { Postsurgical bed after } \\
\text { Whipple procedure }\end{array}$ & 4 \\
\hline Multiple sites & 8 \\
\hline \multicolumn{2}{|l|}{ Bleeding artery } \\
\hline Gastroduodenal artery & $17(19.310 \%)$ \\
\hline Hepatic artery & $15(17.45 \%)$ \\
\hline Colic artery & $8(9.09 \%)$ \\
\hline Multiple vessels & $8(9.09 \%)$ \\
\hline Gastric artery & $4(4.54 \%)$ \\
\hline Splenic artery & $3(3.40 \%)$ \\
\hline Other & $8(9.09 \%)$ \\
\hline
\end{tabular}

patients). The technical success rates of embolization for upper GIB and lower GIB were 98.4 and 91.7\%, respectively, and there was no significant difference $(p=0.294)$. The target vessel could not be successfully embolized in one patient with hepatocellular carcinoma and in two patients with duodenal ulcer. These three patients underwent surgical hemostasis successfully. In most of the cases ( $n=81,92.04 \%$ ), a single vessel was embolized; however, in seven patients, multiple vessels were embolized (-Table 3). The embolic materials that were used are listed in - Table 2. Coils were used as the embolic material in majority of cases, either alone ( $n=45 ; 51.1 \%$ ) or in combination with another material $(n=13 ; 14.7 \%)$. In patients with lower GIB, coils alone were used mainly (84\%); however, in those with upper GIB, particles such as Gelfoam and/or polyvinyl alcohol (PVA) particles were the most frequently used embolic materials $(46 \%)(p<0.001)$.

Clinical success was achieved in $81.8 \%(n=72)$ of patients. The clinical success rate was 83.9 and $79.2 \%$ for upper and lower GIB, respectively, and there was no significant difference ( $p=0.438)$. Twelve (13.6\%) patients underwent surgical 
Table 3 Embolization material and sites

\begin{tabular}{|c|c|}
\hline Factors & No. of patients \\
\hline \multicolumn{2}{|l|}{ Site of embolization } \\
\hline Hepatic artery & 29 \\
\hline Right & 11 \\
\hline Left & 8 \\
\hline Common & 8 \\
\hline Right and left & 2 \\
\hline GDA & 16 \\
\hline Colic artery & 9 \\
\hline Multiple sites & 7 \\
\hline Gastric artery & 6 \\
\hline Pancreaticoduodenal artery & 5 \\
\hline SMA & 4 \\
\hline Other & 12 \\
\hline \multicolumn{2}{|l|}{ Materials used } \\
\hline Coil & 45 \\
\hline Gelfoam & 12 \\
\hline Coil and Gelfoam & 10 \\
\hline Coil and PVA particles & 3 \\
\hline Embospheres & 5 \\
\hline PVA particles & 6 \\
\hline n-acetyl cyanoacrylate & 2 \\
\hline Microcoils & 2 \\
\hline Bead blocks & 3 \\
\hline
\end{tabular}

Abbreviations: GDA, gastroduodenal artery; PVA, polyvinyl alcohol; SMA, superior mesenteric artery.

intervention to achieve hemostasis following embolization and three of them died subsequently. In four cases, repeat embolization was required to achieve hemostasis. Ischemic complications following embolization were seen in three (3.4\%) patients of whom two required segmental surgical bowel resection (right colon in one and jejunum in another patient). In the patient with right colon ischemia, coils were used for vasa recta branch embolization, whereas Gelfoam was used for embolization of a jejunal arterial branch in the patient with subsequent jejunum ischemia. Asymptomatic left hepatic lobe ischemia was noted on subsequent imaging in one patient following embolization of a pseudoaneurysm of the proper hepatic artery; this patient responded appropriately to conservative management.

Rebleeding in the first 24 hours after embolization occurred in $14.8 \%$ (13 out of 88 patients). Five underwent surgical management, whereas three had successful repeat angiographic intervention. Overall, four patients with rebleeding in 24 hours died during the hospitalization. Rebleeding rate increased to $18.2 \%$ (16 out of 88 patients) at 30 days. Out of these 16 patients, 6 required surgery and 6 underwent successful repeat angiographic intervention. The 24-hour and 30-day mortality rates were 9.1 and $11.3 \%$, respectively. Rebleeding occurred most commonly in the setting of duodenal ulcer $(n=4)$, trauma $(n=3)$, after Whipple procedure $(n=3)$, gastric ulcer $(n=2)$, and diverticulum $(n=2)$. The mean duration of follow-up was 843.23 days and the overall mortality was $28.4 \%$ ( 25 out of 88 patients). The rates of 24-hour and 30-day rebleeding and survival rates were not statistically different in patients with upper GIB when compared with those with lower GIB. However, the death rate was significantly higher in patients with upper GIB $(p=0.032)$. Although the most frequent cause of death was multifactorial, five patients died as a result of persistent profuse GIB.

None of the initial comorbidities or arteriographic parameters had a substantial influence on the clinical success at 24 hours after embolization. Multivariate analysis showed RBC transfusion requirements within 12 hours after embolization as the only significant factor associated with failed treatment and/or recurrent GIB within 24 hours after the procedure. However, prior GIB was an independent variable associated with 30-day rebleeding $(p=0.026)$. These results are summarized in -Table 4.

\section{Discussion}

This report represents clinical and technical effectiveness of angiography and transcatheter intervention in patients with nonvariceal GIB. In this retrospective study, multivariate analyses identified $\mathrm{RBC}$ transfusion requirements within the first 12 hours after embolization and prior GIB as significant factors associated with failed treatment/recurrent GIB within 24-hour and 30-day after the embolization, respectively. Our findings complement those of a recent study providing additional insights into the short-term outcomes of angiographic therapy and subsequent embolization in patients with GIB. ${ }^{11-13}$

Management options for patients with acute nonvariceal upper GIB that does not respond to initial endoscopic treatment include repeat endoscopy, emergency surgery, and angiographic embolization. Several studies have compared the effectiveness of surgery to embolization in treating patients with GIB. ${ }^{14-16}$ In one study, embolization and surgery have shown to result in similar outcomes in patients with endoscopically unmanageable nonvariceal upper GIB. ${ }^{17}$ Defreyne et al showed that the choice of rescue has no significance on the outcome; however, the unsuccessful hemostasis and corticosteroid use negatively affected the outcome. ${ }^{14}$ They suggested that refractory nonvariceal upper GIB and the identification of a peptic ulcer at endoscopy were the determinant factors in deciding for salvage surgery, and arteriography was reserved for pursuit of unclear overt upper GIB. This rationale is likely inspired by the evidence that an unguided emergency laparotomy is unpredictable and risky. Also, results from multiple studies favor embolization in highrisk surgical patients. ${ }^{14}$ Ripoll et al compared transcatheter arterial embolization and surgery for the treatment of bleeding peptic ulcer after endoscopic treatment failure and found no differences between these therapies in the incidence of recurrent bleeding (29 vs. $23.1 \%$ ), the need for additional 
Table 4 Univariate and multivariate analysis for predictive variables for risk of rebleeding 24 hours and 30 days after AE

\begin{tabular}{|c|c|c|c|c|c|c|c|c|}
\hline \multirow[t]{3}{*}{ Variable } & \multicolumn{4}{|c|}{ Rebleeding after $24 \mathrm{~h}$} & \multicolumn{4}{|c|}{ Rebleeding after $30 \mathrm{~d}$} \\
\hline & \multicolumn{2}{|c|}{$\begin{array}{l}\text { Univariate } \\
\text { analysis }\end{array}$} & \multicolumn{2}{|c|}{$\begin{array}{l}\text { Multivariate } \\
\text { analysis }\end{array}$} & \multicolumn{2}{|c|}{ Univariate analysis } & \multicolumn{2}{|c|}{$\begin{array}{l}\text { Multivariate } \\
\text { analysis }\end{array}$} \\
\hline & $\mathrm{HR}$ & $p$-Value & $\mathrm{HR}$ & $p$-Value & HR & $p$-Value & $\mathrm{HR}$ & $p$-Value \\
\hline Age $>65 y$ & 1.077 & 0.894 & 1.00 & 1.00 & 1.256 & 0.648 & 1.068 & 0.921 \\
\hline Gender & 0.411 & 0.247 & 0.208 & 0.101 & 1.356 & 0.556 & 3.992 & 0.370 \\
\hline Comorbidities $^{\mathrm{a}}$ & 0.993 & 0.990 & 0.442 & 0.353 & 0.722 & 0.546 & 0.569 & 0.391 \\
\hline Use of anticoagulant & 0.445 & 0.431 & 2.310 & 0.258 & 1.324 & 0.710 & 1.675 & 0.535 \\
\hline Upper versus lower & 0.874 & 0.974 & 2.237 & 0.588 & 0.378 & 0.301 & 0.229 & 0.089 \\
\hline Coils versus other agent & 0.149 & 0.150 & 0.382 & 0.550 & 0.104 & 0.164 & 1.405 & 0.561 \\
\hline Technical success & 2.361 & 0.480 & 3.849 & 0.409 & 2.581 & 0.802 & 1.690 & 0.985 \\
\hline Prior GIB & 6.526 & 0.072 & 7.740 & 0.065 & 5.629 & 0.003 & 4.149 & 0.026 \\
\hline Transfusion before AE & 0.904 & 0.867 & 1.930 & 0.396 & 1.221 & 0.699 & 1.826 & 0.295 \\
\hline Transfusion after AE & 4.831 & 0.009 & 7.518 & 0.014 & 1.670 & 0.311 & 1.782 & 0.289 \\
\hline
\end{tabular}

Abbreviation: AE, arterial embolization; GIB, gastrointestinal bleeding; HR, hazard ratio.

${ }^{a}$ Comorbidities included diabetes mellitus, stroke, cardiovascular disease, and renal disease.

surgery (16.1 vs. $30.8 \%$ ), or death (25.8 vs. $20.5 \%) .{ }^{16}$ However, it should be noted that the patients who underwent embolization were older and had greater incidences of heart disease and previous anticoagulation treatment. ${ }^{16}$

Recently, Lee et al assessed the efficacy and clinical outcomes of embolization for acute nonvariceal upper GIB with 98\% technical success; however, they reported $47 \%$ rebleeding rate in 30 days. ${ }^{18}$ In this study, we reported a technical success rate of $96.6 \%$ for embolization and an initial clinical success rate of $81.8 \%$; these results reflect the effectiveness and safety of angiographic intervention in the management of nonvariceal GIB and is consistent with the results of a previous study. ${ }^{19}$ of note, our complication rate was lower than that reported previously (3.5\% vs. 6 and 9\%) 11,19 with a 30 -day clinical success rate of $70.5 \%$. We embolized not only the artery that demonstrated signs of bleeding on angiography but also those vessels supplying an area of arterial bleeding documented on endoscopy despite the lack of angiographic evidence of bleeding. ${ }^{20,21}$ This was referred to as empiric embolization and was performed in 25 (28\%) patients. This may be the reason why our study demonstrated higher clinical success compared with prior studies.

In our study, we have evaluated clinical, laboratory, endoscopy, and angiography data. This information enabled us to incorporate different prognostic factors into the multivariate analysis to evaluate their impact on rebleeding as well as mortality. Lundgren et al provided evidence that the use of anticoagulants or corticosteroids at admission, need for vasopressors before embolization, and use of coils during embolization are all associated with clinical failure of embolization in the upper GIB population. They evaluated the clinical success only immediately after the embolization ${ }^{11}$; however, in our study we provided the 24-hour and 30-day rebleeding rates and the associated factors that would be a robust predictor of the long term outcome. Although several studies have reported coagulopathy as a significant risk factor for rebleeding, 22,23 in the current series it had no significant association with rebleeding risk. In our study, RBC transfusion requirement after embolization was the only independent predictor of rebleeding after 24 hours which is consistent with prior studies. ${ }^{14,15}$ Defreyne et al reported that patients experiencing early rebleeding had received significantly higher amounts of packed cells, although there was no significant difference in terms of hemoglobin concentration when compared with those who had successful embolization. ${ }^{23}$ There was no significant association between the method of embolization and the embolic materials used in regard to the failure rate. Also, there was no significant difference between various initial bleeding sites and their association with rebleeding. In our study, coils were used as the sole embolic material in majority of cases (51.1\%). The clinical success was comparable to that of using other embolic materials with or without coils. This contrasts with prior studies showing that the use of coil as the only embolic material can be associated with an increased risk of clinical failure. ${ }^{11}$ It is possible that the current generation of coils used in this study has higher thrombogenicity compared with those used in prior studies. Additionally, technical advances in coil design might have allowed better packing of the coils during embolization procedure, leading to high technical and clinical success with coil embolization. We had 24 hours rebleeding rate of $14.8 \%$. Considering the fact that the patients who usually undergo embolization for GIB are usually highrisk patients with several comorbidities that already have failed standard medical management, rebleeding rate of below $15 \%$ in this subgroup of patient can still be considered clinically acceptable. However, improvement of the techniques is required to optimize the outcomes. Larger studies with subgroups of patients with different comorbidities is 


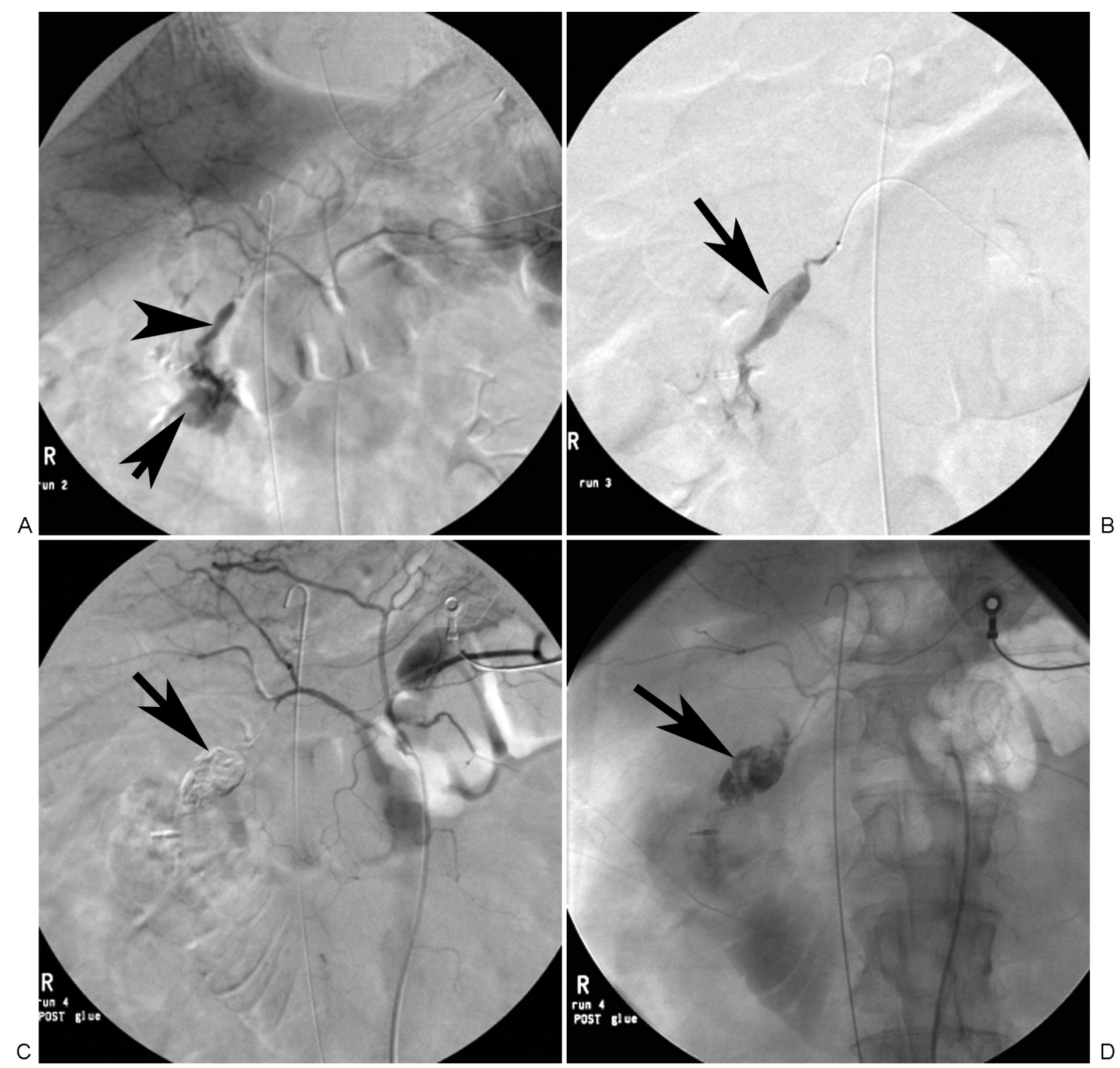

Fig. 1 A 55-year-old woman with upper gastrointestinal bleeding secondary to duodenal ulcer. (A) Celiac arteriogram shows a pseudoaneurysm (arrowhead) of the gastroduodenal artery with associated active extravasation (arrow) in to the duodenum. (B) Selective gastroduodenal arteriogram again identifies the pseudoaneurysm (arrow). The aneurysm was treated with n-acetyl cyanoacrylate. (C,D) Postembolization angiogram demonstrates no more perfusion of the aneurysm. The cast (arrow) of n-acetyl cyanoacrylate can be seen.

required to suggest which group of patients will benefit more from embolization for GIB.

Studies have shown that rebleeding after 3 days is more likely to be related to clinical factors than to the embolization technique. Malignancy, vasculitis, vigorous hypovolemic shock therapy, and high quantities of packed cell transfusions before embolization (mean 9.5 units per 48 hours) had a significantly higher risk for 30-day rebleeding. ${ }^{24}$ However, in our cohort of patients, prior GIB was the only independent variable associated with 30 -day rebleeding. This may be explained by the fact that only 16 patients in our study required transfusion of more than 10 units of blood and we had no cases of vasculitis.

Upper gastrointestinal tract has been reported as a highrisk region for early failure. ${ }^{24}$ In our patients, the rebleeding risk was similar in both upper and lower GIB, although the death rate was significantly higher for those with upper GIB. Hur et al assessed the safety and the efficacy of transcatheter arterial embolization for lower GIB and reported a technical success rate of $96.4 \%$, clinical success rate of $75.0 \%$, recurrent bleeding rate of $17.4 \%$, and major complication rate of $4.6 \%{ }^{25}$ In our study, we had comparable results: the technical success was 98.4 and $91.7 \%$ and the clinical success rate was 83.9 and $79.2 \%$ for upper and lower GIB, respectively.

Although endoscopy is considered to be the first-line diagnostic modality in the setting of acute GIB, endoscopy often fails to depict the exact focus of bleeding when excessive blood or clots impair visualization. Recently, the use of CT angiography as a first-line modality in the detection and localization of GIB has increased. ${ }^{26}$ Ren et al recently 
evaluated the value of CT angiography for diagnosis and therapeutic planning in lower GIB and demonstrated that CT angiography detected active extravasation of contrast material in 57 patients with an accuracy of $90.5 \%$ in the detection of acute lower GIB. ${ }^{27}$ Another study evaluated the role of negative CT angiography in patient management, $20.6 \%$ of patients presenting with upper GIB had positive CT scan while $32.2 \%$ were positive in patients with lower GIB and concluded that patients with upper GIB who had negative CT angiography usually require further intervention to stop the bleeding. 28

Our study has several limitations. It is retrospective, is limited to a single-center, and focuses on short-term outcomes. A larger, multicenter prospective study with longer follow-up is required. For mortality measures, we assessed all-cause mortality for our patients. GIB-related mortality would have been a better primary end point but this was hard to specify in some patients with several comorbidities. Accordingly, we chose to report and correlate all-cause mortality in our study. We also included patients with different underlying causes for GIB in the multivariate analysis to identify predictive factors for outcomes. One might argue that the nature of the underlying cause for the vessel injury leading to the GIB may play an important role in the outcomes; for example, diffuse tumoral vessel bleeding has a different clinical course than does a bleeding ulcer. The relatively small number of patients in our cohort limited our ability to stratify risks based on the nature of the hemorrhage. Additionally, as the results of CT angiography were available in a small number of patients in our cohort, the findings were not included in the analysis.

\section{Conclusion}

Endovascular embolization has an acceptable clinical success and a high technical success with minimal complications for treating patients presenting with nonvariceal GIB. Embolization is equally effective in the management of upper and lower GIB. In patients with early rebleeding, repeat intervention may increase the overall clinical success.

\section{Author Contribution}

Azadeh Elmi contributed in study concept, acquisition of data, analysis and interpretation of data, drafting of the manuscript, and statistical analysis. T. Gregory Walker, MD, Suvranu Ganguli, and Sanjeeva P. Kalva contributed in study concept and design, acquisition of data, critical revision of the manuscript for important intellectual content, administrative, technical, and material support, and study supervision.

\section{References}

1 Rockey DC. Gastrointestinal bleeding. Gastroenterol Clin North Am 2005;34(4):581-588

2 Laing CJ, Tobias T, Rosenblum DI, Banker WL, Tseng L, Tamarkin SW. Acute gastrointestinal bleeding: emerging role of multide- tector CT angiography and review of current imaging techniques. Radiographics 2007;27(4):1055-1070

3 Ferguson CB, Mitchell RM. Nonvariceal upper gastrointestinal bleeding: standard and new treatment. Gastroenterol Clin North Am 2005;34(4):607-621

4 Welch CE, Hedberg S. Gastrointestinal hemorrhage. I. General considerations of diagnosis and the rapy. Adv Surg 1973; 7:95-148

5 Ang D, Teo EK, Tan A, et al. A comparison of surgery versus transcatheter angiographic embolization in the treatment of nonvariceal upper gastrointestinal bleeding uncontrolled by endoscopy. Eur J Gastroenterol Hepatol 2012;24(8):929-938

6 Arrayeh E, Fidelman N, Gordon RL, et al. Transcatheter arterial embolization for upper gastrointestinal nonvariceal hemorrhage: is empiric embolization warranted? Cardiovasc Intervent Radiol 2012;35(6):1346-1354

7 Wong TC, Wong KT, Chiu PW, et al. A comparison of angiographic embolization with surgery after failed endoscopic hemostasis to bleeding peptic ulcers. Gastrointest Endosc 2011;73(5):900-908

8 van Vugt R, Bosscha K, van Munster IP, de Jager CP, Rutten MJ. Embolization as treatment of choice for bleeding peptic ulcers in high-risk patients. Dig Surg 2009;26(1):37-42

9 Loffroy R, Guiu B, Cercueil JP, et al. Refractory bleeding from gastroduodenal ulcers: arterial embolization in high-operativerisk patients. J Clin Gastroenterol 2008;42(4):361-367

10 Kuo WT, Lee DE, Saad WE, Patel N, Sahler LG, Waldman DL. Superselective microcoil embolization for the treatment of lower gastrointestinal hemorrhage. J Vasc Interv Radiol 2003;14(12): 1503-1509

11 Lundgren JA, Matsushima K, Lynch FC, Frankel H, Cooney RN. Angiographic embolization of nonvariceal upper gastrointestinal bleeding: predictors of clinical failure. J Trauma 2011;70(5): 1208-1212

12 Hongsakul K, Pakdeejit S, Tanutit P. Outcome and predictive factors of successful transarterial embolization for the treatment of acute gastrointestinal hemorrhage. Acta Radiol 2014;55(2): 186-194

13 Rossetti A, Buchs NC, Breguet R, Bucher P, Terraz S, Morel P. Transarterial embolization in acute colonic bleeding: review of 11 years of experience and long-term results. Int J Colorectal Dis 2013;28(6):777-782

14 Defreyne L, De Schrijver I, Decruyenaere J, et al. Therapeutic decision-making in endoscopically unmanageable nonvariceal upper gastrointestinal hemorrhage. Cardiovasc Intervent Radiol 2008;31(5):897-905

15 Millward SF. ACR Appropriateness Criteria on treatment of acute nonvariceal gastrointestinal tract bleeding. J Am Coll Radiol 2008; 5(4):550-554

16 Ripoll C, Bañares R, Beceiro I, et al. Comparison of transcatheter arterial embolization and surgery for treatment of bleeding peptic ulcer after endoscopic treatment failure. J Vasc Interv Radiol 2004; 15(5):447-450

17 Jailani RF, Kosai NR, Yaacob NY, et al. Transarterial angioembolization versus surgery after failed endoscopic therapy for nonvariceal upper gastrointestinal bleeding. Clin Ter 2014;165(6): 294-298

18 Lee HH, Park JM, Chun HJ, Oh JS, Ahn HJ, Choi MG. Transcatheter arterial embolization for endoscopically unmanageable non-variceal upper gastrointestinal bleeding. Scand J Gastroenterol 2015; 50(7):809-815

19 Loffroy R, Rao P, Ota S, De Lin M, Kwak BK, Geschwind JF. Embolization of acute nonvariceal upper gastrointestinal hemorrhage resistant to endoscopic treatment: results and predictors of recurrent bleeding. Cardiovasc Intervent Radiol 2010;33(6): 1088-1100

20 Morris DC, Nichols DM, Connell DG, Burhenne HJ. Embolization of the left gastric artery in the absence of angiographic extravasation. Cardiovasc Intervent Radiol 1986;9(4):195-198 
12 Endovascular Embolization in Nonvariceal GIB Elmi et al.

21 Lang EV, Picus D, Marx MV, Hicks ME, Friedland GW. Massive upper gastrointestinal hemorrhage with normal findings on arteriography: value of prophylactic embolization of the left gastric artery. AJR Am J Roentgenol 1992;158(3):547-549

22 Poultsides GA, Kim CJ, Orlando R III, Peros G, Hallisey MJ, Vignati PV. Angiographic embolization for gastroduodenal hemorrhage: safety, efficacy, and predictors of outcome. Arch Surg 2008;143(5):457-461

23 Loffroy R, Guiu B, D'Athis P, et al. Arterial embolotherapy for endoscopically unmanageable acute gastroduodenal hemorrhage: predictors of early rebleeding. Clin Gastroenterol Hepatol 2009; 7(5):515-523

24 Defreyne L, Vanlangenhove P, De Vos M, et al. Embolization as a first approach with endoscopically unmanageable acute nonvariceal gastrointestinal hemorrhage. Radiology 2001;218(3):739-748
25 Hur S, Jae HJ, Lee M, Kim HC, Chung JW. Safety and efficacy of transcatheter arterial embolization for lower gastrointestinal bleeding: a single-center experience with 112 patients. J Vasc Interv Radiol 2014;25(1):10-19

26 Artigas JM, Martí M, Soto JA, Esteban H, Pinilla I, Guillén E. Multidetector CT angiography for acute gastrointestinal bleeding: technique and findings. Radiographics 2013;33(5):1453-1470

27 Ren JZ, Zhang MF, Rong AM, et al. Lower gastrointestinal bleeding: role of 64-row computed tomographic angiography in diagnosis and therapeutic planning. World J Gastroenterol 2015;21(13): 4030-4037

28 Chan V, Tse D, Dixon S, et al. Outcome following a negative CT angiogram for gastrointestinal hemorrhage. Cardiovasc Intervent Radiol 2015;38(2):329-335 